\title{
A Review Study of Information Systems
}

\author{
Forat Falih Hasan \\ Dept. of computer T.Engineering \\ Alkitab University College Kirkuk, Iraq
}

\begin{abstract}
The main objective of this paper is to provide a clear view of the information systems denoted as IS by quick and clear approach starting from the definition, dimension, infrastructure and the main types of information systems. This study creates a spotlight to understand and develop the IS in the future in order to improve the performance of the entire systems. As a result, the management of IS plays a significant role in the success of an organization's performance through a set of procedures and functions.
\end{abstract}

\section{Keywords}

Information, Information systems, Types of IS, Information technology.

\section{INTRODUCTION}

Information systems are considered the most important field so that all modern studies focuses on this area and the organizations compete to realize novel technologies, facilities to support this field. The generator part of this area is the information that was evaluated as the soul of these systems. Hereby, high performance achievement needs to identify the types of IS and the way of work.

The technology plays a great role in information systems' life cycle starting from database (DB) technology that defines as a collection of information or a group of facts in a structured way. Principally, DB stores the current and future data to get them available at any time to be used in the organizations [1]. In the other hand, data warehousing technology is employed to store the data from organization sources externally, internally. Moreover, past and current data helps to provide a great base in order to support decision making processes with respect to the information needed for this purpose at any time accordingly [2].

The data analysis technologies like decision tree, clustering, classification, OLAP and neural network etc., are considered as the big wealth used in a wide range by the international organizations due to the facilities provided to apply decision making processes and to save the time and the cost by intelligent ways [3].
All the above discussions realize reliable information to enhance performances and move the organization to the universal level. Hence, the responsibility of all the kinds of information systems inside the organization such as executive information systems, decision support systems, management information systems and transaction processing systems are assigned to follow the rules in the correct ways to achieve the common objective in the working environment.

To be more specific, this paper provides general and basic evidence regarding the information systems such that the first section defines the information while the other sections give basic information about types of IS.

\section{INFORMATION SYSTEMS}

Information systems (IS) is an integrated environment of the hardware, software, peoples which mainly functions for the purpose of collecting and processing data to valuable information by applying a list of procedures on data collection, thus information is derived from data by IS procedures, and clears the difference between data and information. The data is raw materials and the information is the resultant data of processing [4].

According to the explanation above, the key reasons of converting data to information are:

1- Helps in decision making procedures.

2- Classified as strongest element in an organization.

3- Provides competition values.

4- Enhances structural illustrations.

5- Minimize the level of risks in an organization.

6- Renews the level of knowledge.

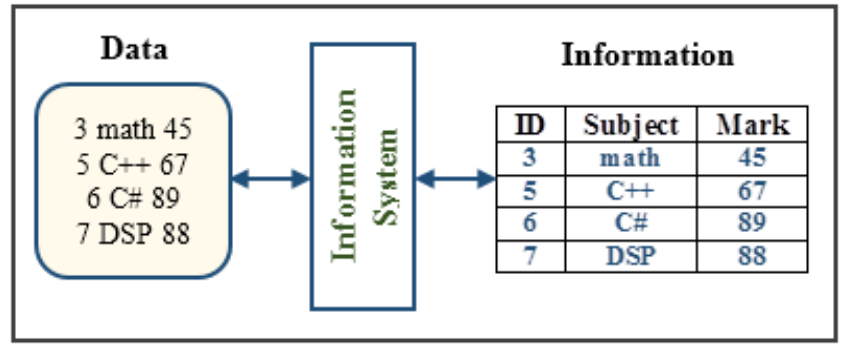

Fig 1: Data and Information 


\subsection{Attributes of the Information}

Information is an ultimate requirement to elevate the prosperity of an organization. The quality expectations of an organization can be specified by decision making processes sensibly. Superior classed information that located in an appropriate framework with respect to the time can illustrate the problems and the chances to solve them in progress. Thus information has certain attributes to increase its utility. For more clarity the attributes are classified as follows [5]:

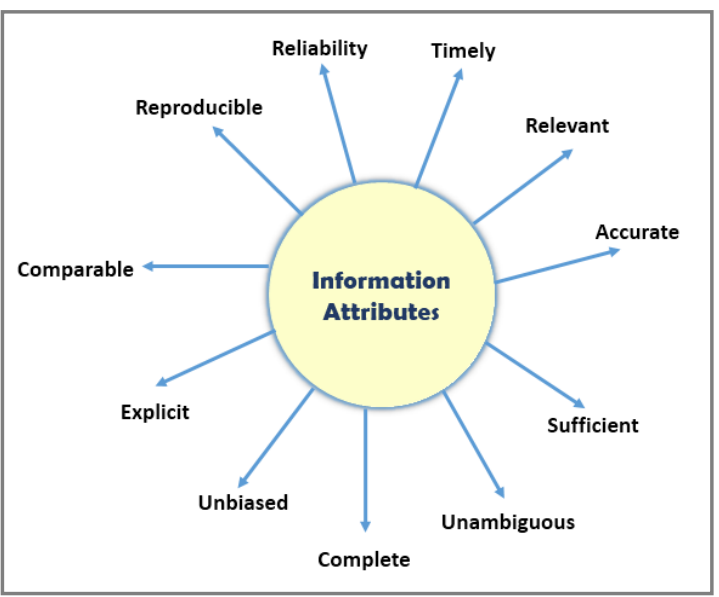

Fig 2: Information Attributes

\subsection{Classification of Information}

The information can be categorized into different ways to activate the direct and the simple awareness to appreciate. The figure 3 below shows the classification of the information [5]:

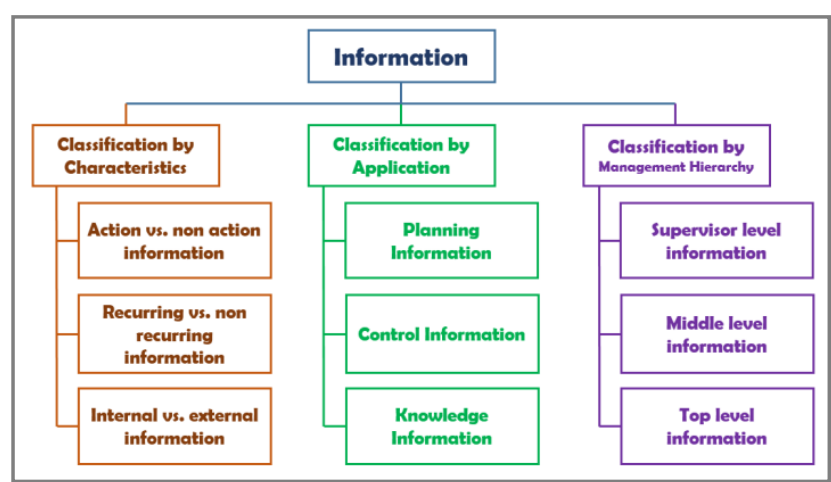

Fig 3: Classification of Information

1- Action vs. non action information: Action information is active information that causes an activity or operation, while the information that communicates only when the status is applied without any operation is called non-action.

2- Recurring vs. non recurring information: The information that is generated in regular intervals is called recurring information, whereas non-repetitive in nature is called non-recurring information.

3- Internal vs. external information: all information that produced from internal sources of any organization is called internal information, though all information that produced from external sources of any organization is called external information.

4- Planning information: this kind of information is used to set up rules and norms for the strategic, tactical and operational planning of the organization activities.
5- Control information: the information collected for the purpose of describing the specific or general situation in the organization activities is called control information.

6- Knowledge information: all kinds of information that is acquired through experience and learning.

Based on Anthony's, from the view of management classification of information that is gathered for the purpose of decision making can be classified as shown in figure 4 below:

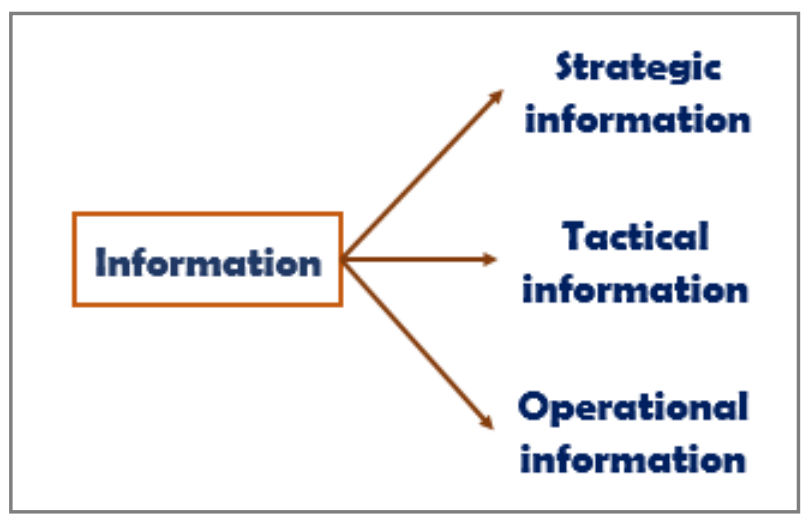

Fig 4: Types of Information Based On Decision Making

All the information collected for the purpose of long term planning is concerned with strategic planning called strategic information. The type of tactical information is used for medium or short term planning collected for achieving activities in very short time around day, week, month or a year is called operational information.

\section{DIMENSIONS OF IS}

To get a complete view of the information systems, the dimensions should be defined clearly for the organization, management, and information technology. A successful information systems produces an excellent value for the firm alongside with a competition value. These dimensions can be explained as follows [6]:

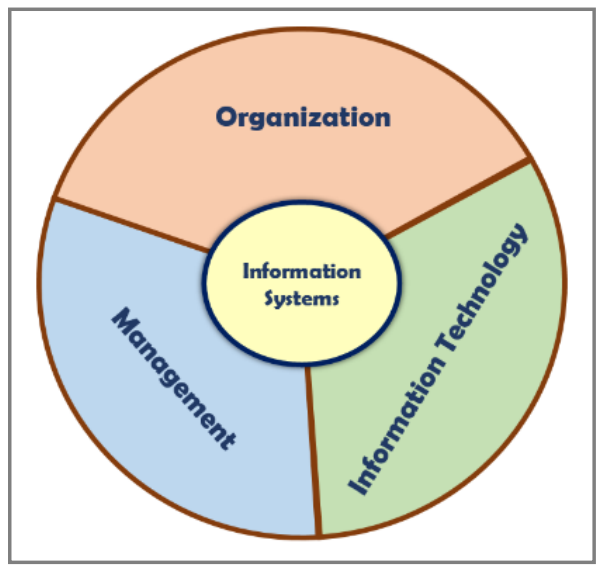

Fig 5: Information systems are more than computers

1- Organization dimension: Information systems are part of organizations. IS will have the standard operating procedure that is the culture of an organization which imbed them. The organization dimension of information systems involves the organization's hierarchy, functional specialties, business process, culture, and political interest 
groups.

2- Management dimension: The management dimension of information systems involves leadership, strategy, and management behavior. The role of information systems is to provide the information needed by the managers to run all the activities by the right ways.

3- Information technology dimension: The technology dimensions consist of computer hardware, software, data management technology, and networking/telecommunications technology (including the internet).

\section{IS INFRASTRUCTURE}

information system infrastructure consists of the integrated environment from the hardware, software, human resources, services, data, knowledge, facilities, and communication all these components are considered a base platform that information system uses to achieve the organizational goals, the figure 6 shows the main components of IS infrastructure[4]:

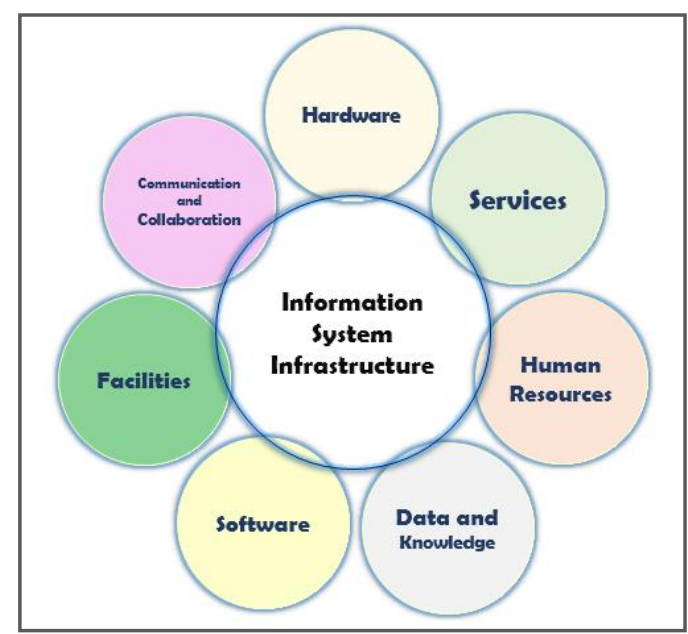

Fig 6: Information System Infrastructure

\section{TYPES OF IS}

The common classification of the information systems based on the use of such systems can be in the fourth levels starting from the top level at executive information systems, the middle level that deals with decision support systems and management information systems to the low level that is concerned with transaction processing systems as shown in the figure below [7]:

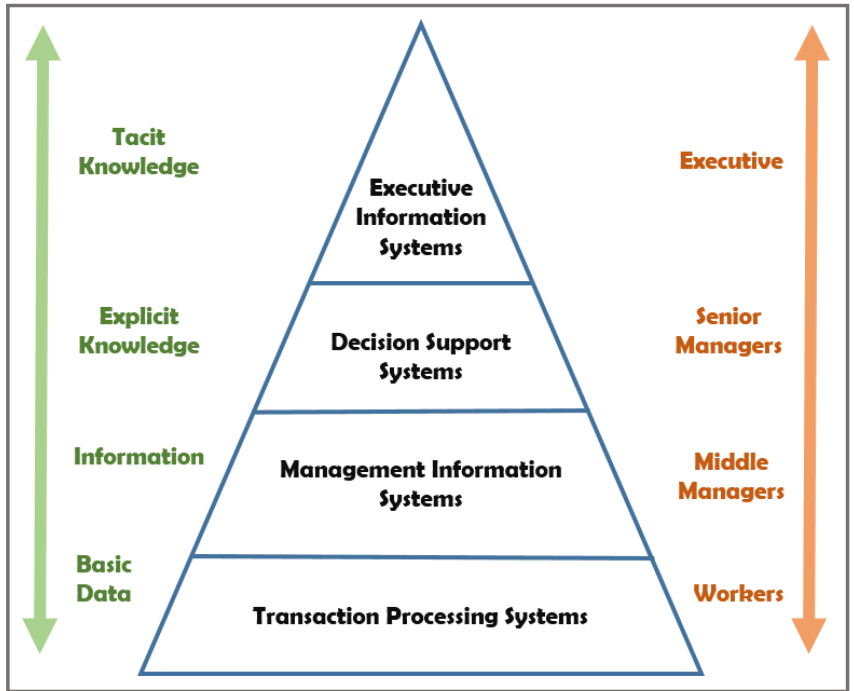

Fig 7: Types and levels of information systems

\subsection{Executive Information Systems (EIS)}

EIS is a system located at the top level of administration pyramid, employed for providing a suitable environment to flow the information from the internal and external sources to meet requirements of the senior executives from the information by providing a framework for accessing easily to the information and achieve the strategic goals of the organization [8].

\subsubsection{Outlines of EIS}

1. Provides a suitable environment to easy access the internal and external information.

2. Provides easy access to data warehousing system that contains the past and latest data.

3. Concerned with high level management.

4. Uses data mining techniques for the purpose of aggregate, analysis data and predicting the future.

5. Provides a better view using both graphics and text tools for the purpose of reporting the situations and explaining the status.

\subsection{Decision support systems (DSS)}

DSS is a computer-based application used to collect and analyze data for the purpose of presenting information in an easy way to make a better decision and solve business problems [9].

\subsubsection{Outlines of DSS}

1. Helps in future predicting.

2. Supports decision in each phase of the decision process.

3. Helps to determine and solve problems.

The decision making process can be separated into four stages as shown in figure 8 : 


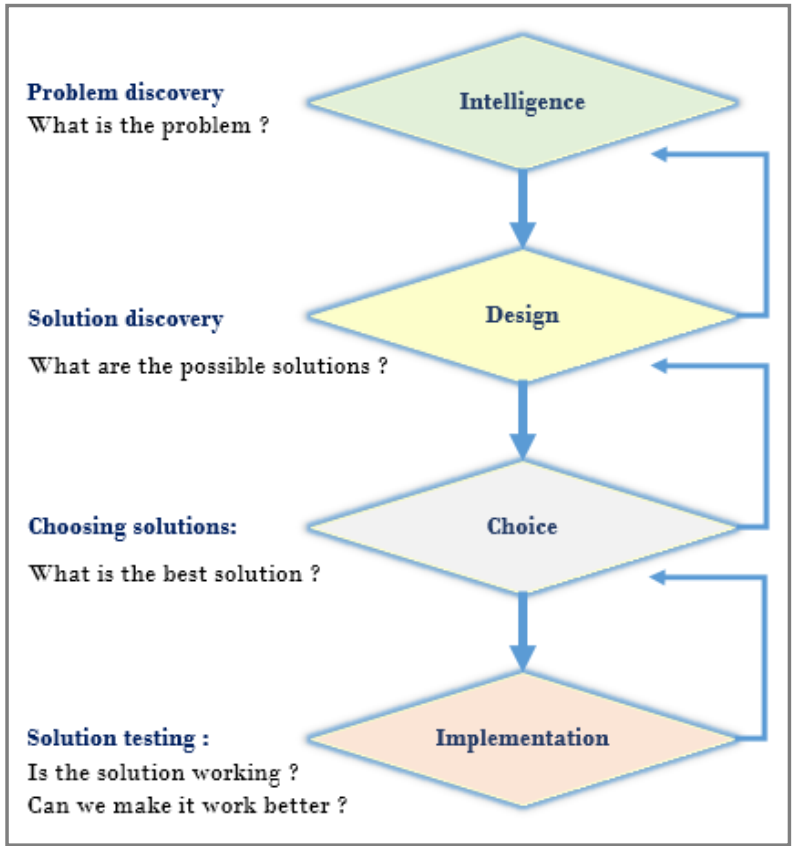

Fig 8: Stages in decision making [10]

\subsection{Management information systems (MIS)}

The structure of MIS consists of three essential components specified as management, information, and systems, which all connected for the purpose of supporting the entire organization parts by the information. Hence, MIS is defined as a system that provides the information for decision making by using organization databases [11].

\subsubsection{Outlines of MIS}

1. Makes information available for all the levels in the organization.

2. Provides information for decision making.

3. Helps to analyze and solve problems.

4. MIS plays an important role in the organization by the functions and procedures that are performed at the entire levels in the organization.

5. Helps in setting planning policies for the organization.

\subsection{Transaction processing systems (TPS)}

TPS is a system located at the low level at the information systems pyramid. TPS is used for gathering, organizing, and storing the data transactions of the organization and make it ready to use by the other information systems in the organization [7].

\subsubsection{Outlines of TPS}

1. Supports all the systems in the organization by the information.

2. Collets the data from the internal and external sources.

3. Used by operational personnel and supervisory levels.

\section{WHY MIS}

MIS plays a vital role in the organization environment due to its responsibility to receive the data from different database resources including the external and internal sources and convert them to useful information for different types and levels in the organization.

Due to the critical position of MIS at the middle of administrative pyramid, the whole system is considered a connection link between the high and low level management, therefore the information flows from the top to bottom and vice versa. The information achieves by the MIS to determine the time and the types, which are the part that must be available to be used by the other information systems.

MIS helps in policy making such as the strategic and tactic policies and enhances the performance, planning, decision making process, choosing among alterative and quality control. Hereby MIS adds a competition value for the organization. The figure 9 shows how the MIS consider a connection link between the other types of information systems and data sources:

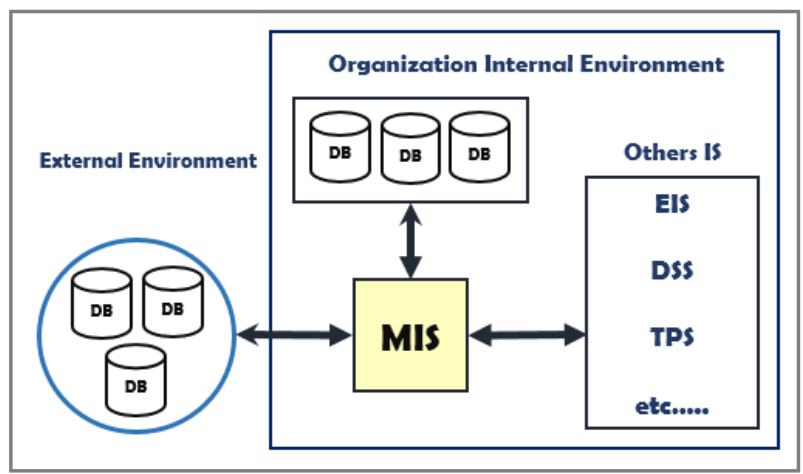

Fig 9: MIS and data sources

Based on the discussion above, MIS is considered as the key reason to success the performance of the information systems; therefore a successful MIS is realized by preparing a suitable environment to work properly, such as software, hardware and people, which all are the factors that have a direct effect on the performance of MIS.

\section{CONCLUSION}

In this paper, we presented the overall facts about information systems by a quick and clear surfing methodology. For more clarity, it is intended to show the following results:

1. The information is considered as the pulses move inside the heart of IS and gives the soul to the organization to survive that is the MIS has got the excessive role to manage these pulses.

2. The information plays a great role to give a competition value to the organization.

3. The improvement of IS performances have a great relation with the MIS in the organization due to the procedures and functions performed regularly.

4. Currently, there is no success without using new and updated technologies.

5. As a future enhancement to this work, studying each type of information systems in details.

\section{AUTHOR'S PROFILE}

Forat Falih Hasan: Assistant Lecturer in Alkitab University College, Iraq. Interested Area (Database, Data Mining and Information System, BI).

\section{REFERENCES}

[1] Ivana Lieskovská Drábiková, Karol Matiaško, Anton Lieskovský,: "Database Object Dependencies Modeling" Software Engineering and Service Science (ICSESS), 7th IEEE International Conference on 23, March, 2017.

[2] K. Dhanasree, C. Shobabindu,: "A survey on OLAP" Computational Intelligence and Computing Research 
(ICCIC), IEEE International Conference on 08, May, 2017.

[3] Rahma Djiroun, Kamel Boukhalfa, Zaia Alimazighi, Faten Atigui, Sandro Bimonte,: "A data cube design and construction methodology based on OLAP queries" IEEE/ACS 13th International Conference of Computer Systems and Applications (AICCSA), pp. 1-8, 2016.

[4] Kodrat Iman Satoto, Kurniawan Teguh Martono, R. Rizal Isnanto,: "Design of management information systems research, publications and community service" Information Technology, Computer, and Electrical Engineering (ICITACEE), 2nd International Conference on 16-18, Oct, 2015.

[5] Tutorials point, simply easy learning. [Online].Available: https://www.tutorialspoint.com/management_informatio $\mathrm{n} \_$system/classification_of_information.htm, (27/12/2017).

[6] Slide share, in slide share. [Online]. Available: https://www.slideshare.net/suleymans19/threedimensions-of-information-systems, (26/12/2017).

[7] Chris kimble, the personal website of Chris kimble. [Online]. Available: http://www.chris-
kimble.com/Courses/World_Med_MBA/Types-ofInformation-System.html, (26/12/2017).

[8] Dr Mir Mohammad Azad, Mohammad Bin Amin, Md Alauddin,: "Executive Information System" IJCSNS International Journal of Computer Science and Network Security, VOL.12 No.5, May, 2012.

[9] Fotis Kitsios, Maria Kamariotou,:"Decision Support Systems and Business Strategy: A Conceptual Framework for Strategic Information Systems Planning" IT Convergence and Security (ICITCS), 6th International Conference on 26-26, Sept, 2016.

[10] Kenneth C. Laudon, Jane P. Laudon,: "Management Information Systems" Management The Digital Firm, Tenth Edition, PHI Learning Private Limited, New delhi, 110001, 2009.

[11] Yousef A.Baker El-Ebiary, Najeeb Abbas AlSammarraie, Yazeed Al Moaiad,: "The impact of Management Information System in educational organizations processes" e-Learning, e-Management and e-Services (IC3e), IEEE Conference on 10-12, Oct, 2016. 